BMJ Open Diabetes Research \& Care

\title{
Comparing the efficacy and safety of insulin detemir versus neutral protamine hagedorn insulin in treatment of diabetes during pregnancy: a randomized, controlled study
}

\author{
Jing ji, ${ }^{1}$ Zhangya He, ${ }^{2}$ Zhao Yang, ${ }^{3}$ Yang Mi, ${ }^{1}$ Na Guo, ${ }^{1}$ Hong Zhao, ${ }^{1}$ Jiayi Gao, ${ }^{2}$ \\ Zhengfeei Ma, ${ }^{4}$ Xiaoqin Luo (D) ${ }^{2}$ Zhen $\mathrm{Han}^{3}$
}

To cite: ji J, He Z, Yang Z, et al. Comparing the efficacy and safety of insulin detemir versus neutral protamine hagedorn insulin in treatment of diabetes during pregnancy: a randomized, controlled study. BMJ Open Diab Res Care 2020;8:e001155. doi:10.1136/ bmjdrc-2019-001155

Received 25 December 2019 Revised 1 February 2020 Accepted 3 March 2020

Check for updates

(c) Author(s) (or their employer(s)) 2020. Re-use permitted under CC BY-NC. No commercial re-use. See rights and permissions. Published by BMJ.

${ }^{1}$ Department of Obstetrics and Gynecology, Northwest Women's and Children's Hospital, Xi'an, China ${ }^{2}$ Department of Nutrition and Food Safety, School of Public Health, Xi'an Jiaotong University, Xi'an, China ${ }^{3}$ Department of Obstetrics and Gynecology, the First Affiliated Hospital of Xi'an Jiaotong University, Xi'an, China ${ }^{4}$ Department of Health and Environmental Sciences, Xi'an Jiaotong-Liverpool University, Suzhou, China

Correspondence to Dr Xiaogin Luo; luoxiaoqin2012@mail.xjtu.edu. cn and Dr Zhen Han; hanzhen@xjtufh.edu.cn

\section{ABSTRACT}

Objective To compare the efficacy and safety of insulin detemir (IDet) versus neutral protamine Hagedorn (NPH) insulin used in pregnant women with diabetes.

Research design and methods A randomized study was conducted in diabetic pregnant women $(n=240)$ (including 132 with pregestational diabetes and 108 with gestational diabetes). All patients were randomly divided into two groups: IDet group $(n=120)$ treated with IDet plus short acting insulin Novolin-R before three meals (RRR-IDet plan), and NPH group treated with NPH plus Novolin-R before three meals (RRR-NPH plan). Patients were enrolled during 12-28 gestation weeks and followed up until delivery.

Results Basal characteristics, such as age, enrollment gestational weeks, average $\mathrm{HbA1c}$, fasting plasma glucose (FPG) and oral glucose tolerance test (OGTT) were similar between two groups. After 1 week of treatment, the FPG of IDet group were significantly lower than NPH group $(p<0.05)$ and the time required to reach the targeted blood glucose level was significantly shorter $(p<0.001)$. After 3 months of treatment, the HbA1c level in the two groups was normalized but there was no significant difference in $\mathrm{HbA1c}$ level. Maternal and neonatal outcomes were comparable between the two therapeutic approaches; however, the incidence of hypoglycemia in IDet group was remarkably lower than that of NPH group $(p<0.05)$. The adverse drug reactions were rare and similar between the two groups.

Conclusions For the treatment of gestational diabetes, both RRR-IDet plan and RRR-NPH plan were reported to control blood glucose effectively. Compared with NPH, IDet could control blood glucose and reached the targets faster and more effectively, thus reducing the number of insulin injections and the incidence of hypoglycemia in pregnant women without increasing adverse birth outcomes. Therefore, for pregnant women with gestational diabetes, who require insulin therapy, IDet would be an ideal basal insulin being worthy of promotion in clinical settings.

\section{INTRODUCTION}

The prevalence of gestational diabetes (GDM) and prepregnant diabetes mellitus

\section{Significance of this study}

What is already known about this subject?

- Hyperglycemia during pregnancy not only leads to the short-term and long-term adverse effects on the mother, but also increases the risk of complications and diseases in the later neonatal period.

- For treatment, insulin detemir (IDet) is currently recommended worldwide. However, its safety and efficiency in pregnant women with diabetes needs to be further studied, especially through clinical trials.

What are the new findings?

- In this controlled clinical trial, we found that, compared with NPH, IDet can control blood glucose and achieve glycemic targets faster and more effectively, and reduce the number of insulin injections and the incidence of hypoglycemia in pregnant women without increasing adverse birth outcomes.

How might these results change the focus of research or clinical practice?

- Considering the outstanding effect of controlling blood glucose and the low risk of adverse outcomes, IDet is an ideal basal insulin for pregnant women with diabetes who need insulin therapy, and it is worth promoting in the clinic.

(PGDM) is increasing year by year, especially for GDM. ${ }^{12}$ In 2017, there were 21.3 million pregnant women who experienced hyperglycemia, of which $86.4 \%$ of them were diagnosed with GDM. ${ }^{3}$ In China, a total of 2.90 million pregnant women suffered from GDM in 2015. ${ }^{4}$ The maternal hyperglycemia not only increases short and longterm adverse effects on mothers, but it is also associated with a higher risk of developing neonatal complications and diseases in their later life. ${ }^{5}$ Hence, for pregnant women with diabetes whose blood glucose cannot be 
controlled through the combination of diet and exercise after 1-2 weeks, insulin is strongly recommended. ${ }^{6}$ Generally, short-acting and ultra-short-acting insulin are prescribed during pregnancy and no long-acting insulin or insulin analogs are preferred clinically. The classic insulin treatment plan is an ultrashort-acting insulin injection immediately before three meals or a shortacting insulin half an hour before three meals (RRR Plan). However, insulin treatment during pregnancy should try to mimic physiology of basal insulin secretion and increased insulin secretion after meals. Therefore, a basal insulin is needed. Neutral protamine Hagedorn $(\mathrm{NPH})$, as a commonly used intermediate-acting basal insulin (before bed and morning), has some issues in clinical applications, such as large absorption variation, short duration of action, significant peak effect, high risk of hypoglycemia, frequent injections, and complicated injection operations. ${ }^{7}$

In recent years, insulin analogs have overcome the limitations of human insulin and are used widely in the management and control of diabetes. ${ }^{8}$ Insulin detemir (IDet) is a long-acting human insulin analog which is newly developed in recent years. In addition to its use in gestational diabetes, IDet improves glycemic control, and is associated with lower rates of hypoglycemia than human insulin. ${ }^{9}{ }^{10}$ In 2012, the US Food and Drug Administration (FDA) and China FDA approved the insulin detemir to treat hyperglycemia during pregnancy. Compared with $\mathrm{NPH}$, the effect of IDet is much closer to physiologic basal insulin secretion with a longer duration and flatter mode (no peaks) of action. However, few studies of observing the safety and efficiency of IDet in Chinese pregnant women with diabetes are reported in the literature. With an increasing incidence of gestational diabetes, the safety and efficiency of IDet during pregnancy is needed to be further studied. Therefore, this study aimed to compare the efficiency and safety between IDet combined with short-acting Novoline R and NPH in combination with Novoline $\mathrm{R}$ in pregnant women with diabetes.

\section{RESEARCH DESIGN AND METHODS \\ Study design}

This randomized study was conducted between January 1st 2014 and December 31st 2015 in the Northwest Women and Children's Hospital (NWCH). This study randomized 240 patients with diabetes developed during pregnancy, including GDM $(\mathrm{n}=108)$ and PGDM $(\mathrm{n}=132)$. All patients were enrolled at gestational age of 12-28 weeks and received health education regarding the fundamental knowledge of gestational diabetes, nutrition and exercise in a 1-day obstetric diabetes clinic. After 1 week of blood glucose monitoring, all patients were evaluated and those who still needed basic-prandial intensive insulin therapy were included. The inclusion criteria were as follows: (1) age $\geq 18$ years; (2) singleton and (3) for GDM patients, the fasting plasma glucose (FPG), 30 min before meals and random blood glucose at night were $>5.3 \mathrm{mmol} / \mathrm{L}$ with blood glucose of 2 hours after meals $>6.7 \mathrm{mmol} / \mathrm{L}$; for PGDM patients, the cut-offs of blood glucose were $>5.6 \mathrm{mmol} / \mathrm{L}$ and $>7.1 \mathrm{mmol} / \mathrm{L}$, respectively. Patients with severe heart, liver and kidney disease or any forms of assisted reproduction were excluded. After screening, we assign subjects random numbers on the basis of patient ID, then eligible patients were randomized to either IDet $(n=120)$ or NPH insulin groups $(n=120)$. IDet group was treated with IDet plus short acting insulin Novolin-R before three meals (RRRDet plan), and NPH group was treated with NPH plus Novolin-R before three meals (RRR-NPH plan). All basal insulin doses were titrated according to FPG or preprandial blood glucose values. Insulin Novolin-R doses were titrated according to preprandial and postprandial blood glucose values. Insulin dose and weight gain during the pregnancy were also calculated.

\section{Endpoints}

The primary endpoint was FPG, preprandial and postprandial blood glucose concentrations after 1 week of treatment. Secondary endpoints included HbAlc, FPG, preprandial and postprandial blood glucose after 3 months of treatment. All insulin doses were adjusted, in accordance with recommendations of the Chinese Guidelines for Diagnosis and Treatment of Pregnancy with Diabetes 2014 $4^{11}$ and were listed as follows: for GDM patients, the FPG and preprandial blood glucose targets were $3.3-5.3 \mathrm{mmol} / \mathrm{L}$, and a 2-hour postprandial blood glucose target was $4.4-6.7 \mathrm{mmol} / \mathrm{L}$; while for PGDM patients, the FPG and preprandial blood glucose targets were $3.3-5.6 \mathrm{mmol} / \mathrm{L}$, and a 2-hour postprandial blood glucose target was 3.6-7.1 mmol/L, respectively. Hypoglycemia was diagnosed when the blood glucose was below the lower limit of target value of each level with or without symptoms of hypoglycemia. Maternal safety endpoints included gestational week, cesarean section, hypoglycemia, gestational hypertension, infection, polyhydramnios and adverse events. ${ }^{12}$ Other maternal endpoints included weight gain during the pregnancy and insulin dose. Neonatal safety endpoints included malformation, premature birth, hypoglycemia, hyperbilirubinemia, low birth weight and macrosomia.

\section{Statistics}

The sample size was calculated based on the assumption that IDet was non-inferior to NPH insulin by more than a prespecified FPG margin of $0.5 \%$. Totally, 120 patients in each group would be needed for $80 \%$ power at the $5 \%$ level of significance. All statistical analyses were performed using SPSS V.23.0 and STATA V.12.0. Data were presented as means \pm SD for continuous variables and as percentages for categorical variables. Twotail unpaired Student's t-tests were used when applicable to compare the pairs of means or longitudinal values. $\chi^{2}$ was performed to compare percentages. Otherwise, the rank sum test was used. $\mathrm{P}$ value $<0.05$ was considered significant. 
Table 1 Baseline characteristics of patients receiving IDet or NPH insulin (means \pm SD)

\begin{tabular}{|c|c|c|c|c|}
\hline Variable & IDet & NPH & $T / \chi^{2}$ & $P$ value \\
\hline Age (years) & $31.67 \pm 4.16$ & $30.84 \pm 5.24$ & 1.35 & 0.178 \\
\hline $\mathrm{BMI}\left(\mathrm{kg} / \mathrm{m}^{2}\right)$ & $24.82 \pm 3.53$ & $24.39 \pm 3.90$ & 0.553 & 0.581 \\
\hline Family history of diabetes, $\mathrm{n}(\%)$ & $29(24.2)$ & $34(28.3)$ & 0.538 & 0.463 \\
\hline Enrollment gestational age (weeks) & $27.69 \pm 6.05$ & $27.70 \pm 5.86$ & 0.011 & 0.991 \\
\hline FPG-OGTT (mmol/L) & $7.16 \pm 1.88$ & $7.38 \pm 2.56$ & 0.775 & 0.44 \\
\hline 1-hour-OGTT glucose (mmol/L) & $13.65 \pm 3.12$ & $14.12 \pm 3.65$ & 1.107 & 0.269 \\
\hline 2-hour-OGTT glucose (mmol/L) & $11.91 \pm 3.62$ & $12.33 \pm 4.20$ & 0.847 & 0.398 \\
\hline HbA1c before treatment (\%) & $6.43 \pm 1.23$ & $6.48 \pm 1.19$ & 0.342 & 0.733 \\
\hline FPG before treatment $(\mathrm{mmol} / \mathrm{L})$ & $6.84 \pm 1.31$ & $6.86 \pm 1.13$ & 0.103 & 0.918 \\
\hline 30 min preprandial blood glucose before treatment $(\mathrm{mmol} / \mathrm{L})$ & $6.96 \pm 1.34$ & $7.03 \pm 1.57$ & 0.356 & 0.722 \\
\hline 2-hour postprandial blood glucose before treatment ( $\mathrm{mmol} / \mathrm{L})$ & $9.40 \pm 1.62$ & $9.55 \pm 1.54$ & 0.741 & 0.459 \\
\hline
\end{tabular}

BMI, body mass index; FPG, fasting plasma glucose; IDet, insulin detemir; NPH, neutral protamine Hagedorn; OGTT, oral glucose tolerance test.

\section{RESULTS}

Table 1 shows the demographics and basal glucose of patients in both the IDet and NPH groups. The main demographics at baseline, such as age, body mass index (BMI), family history of diabetes and gestational age at recruitment were similar between both groups. The blood glucose and HbA1c levels before treatment were also similar between both groups.

\section{Efficiency}

After 7 days of treatment, both the treatments decreased the blood glucose levels while the FPG $(5.33 \pm 0.72$ vs $5.71 \pm 0.87 \mathrm{mmol} / \mathrm{L}$, respectively; $\mathrm{P}<0.001$ ), 30 min preprandial blood glucose $(5.26 \pm 0.70$ vs $5.70 \pm 0.73 \mathrm{mmol} / \mathrm{L}$, respectively; $\mathrm{P}<0.001$ ) and 2-hour postprandial blood glucose $(6.73 \pm 0.79$ vs $7.38 \pm 0.80 \mathrm{mmol} / \mathrm{L}$, respectively; $\mathrm{P}<0.001)$ were much lower in the IDet group than in those in the NPH group. In addition, the declines of the three glycemic indicators mentioned above were more significant in the IDet group than those in the NPH group (all $\mathrm{P}<0.05$ ) (table 2). After 3 months of treatment, there were no significant between-group differences in the levels of HbA1c $(5.66 \% \pm 0.83 \%$ vs $5.80 \% \pm 0.81 \% ; \mathrm{P}=0.191)$ and within-group differences between HbAlc before and after treatment in the two groups $(0.77 \% \pm 1.53 \%$ vs $0.68 \% \pm 1.08 \% ; \mathrm{P}=0.740)$. Within this period of treatment, we also found that the days of FPG and preprandial blood glucose to reach the targeted cut-offs $(4.96 \pm 3.20$ vs $7.06 \pm 2.94$ days; $\mathrm{P}<0.001)$ and days of blood glucose reaching the targeted cut-offs throughout all day $(6.94 \pm 3.99$ vs $10.82 \pm 2.63$ days; $\mathrm{P}<0.001)$ in the IDet group were less than those in the NPH group (table 3 ).

The average needs of IDet $(12.32 \pm 5.05$ vs $9.10 \pm 5.93 \mathrm{u} /$ day; $\mathrm{P}<0.001)$ and the total insulin dose $(47.89 \pm 18.94$ vs $42.21 \pm 17.14 \mathrm{u} /$ day; $\mathrm{P}=0.016$ ) in the IDet group were higher than those in the NPH group, but the frequency of insulin injections in the IDet group was less than that in the NPH group $(3.74 \pm 0.78$ vs $4.15 \pm 0.36 \mathrm{t} /$ day; $\mathrm{P}<0.001)$. There was no difference in the dose of Novolin-R between the IDet and NPH groups $(35.57 \pm 16.27$ vs $33.11 \pm 14.23 \mathrm{u} /$ day, respectively; $\mathrm{P}=0.216$ ) (table 4 ).

\section{Safety}

Table 5 shows the maternal safety endpoints between IDet and NPH group. As presented, there was no difference in the incidence of pregnancy outcomes, including gestational age, cesarean section, hypertension during pregnancy, polyhydramnios and infection between both groups $(\mathrm{P}>0.05)$. There was also no difference in weight gain during pregnancy between both groups $(12.21 \mathrm{~kg}$ in

Table 2 Comparison of blood glucose levels and blood glucose decrease after 7 days of treatment in the IDet and NPH groups (means $\pm \mathrm{SD}$ )

\begin{tabular}{lllll}
\hline Blood glucose after 7 days of treatment & IDet & NPH & $\boldsymbol{T}$ & P value \\
\hline FPG (mmol/L) & $5.33 \pm 0.72$ & $5.71 \pm 0.87$ & 3.609 & $<0.001$ \\
30 min preprandial blood glucose (mmol/L) & $5.26 \pm 0.70$ & $5.70 \pm 0.73$ & 4.644 & $<0.001$ \\
\hline 2-hour postprandial blood glucose (mmol/L) & $6.73 \pm 0.79$ & $7.38 \pm 0.80$ & 6.381 & $<0.001$ \\
FPG decrease (mmol/L) & $1.51 \pm 1.41$ & $1.15 \pm 0.83$ & 2.386 & 0.018 \\
\hline 30 min preprandial blood glucose decrease (mmol/L) & $1.70 \pm 1.38$ & $1.34 \pm 1.41$ & 2.021 & 0.044 \\
2-hour postprandial blood glucose decrease (mmol/L) & $2.66 \pm 1.65$ & $2.17 \pm 1.59$ & 2.308 & 0.022 \\
\hline
\end{tabular}

FPG, fasting plasma glucose; IDet, insulin detemir; NPH, neutral protamine Hagedorn. 
Table 3 Comparison of the efficiency between the IDet and NPH groups after 3 months of treatment (means \pm SD)

\begin{tabular}{llrrr}
\hline Variable & IDet & NPH & T/Z & P value \\
\hline HbA1c (\%) & $5.66 \pm 0.83$ & $5.80 \pm 0.81$ & 1.313 & 0.191 \\
Difference of HbA1c (\%) & $0.77 \pm 1.53$ & $0.68 \pm 1.08$ & 0.331 & 0.740 \\
Days of FPG and preprandial blood glucose reaching the target (day) & $4.96 \pm 3.20$ & $7.06 \pm 2.94$ & 5.294 & $<0.001$ \\
Days of blood glucose reaching the target throughout all day (day ) & $6.94 \pm 3.99$ & $10.82 \pm 2.63$ & 8.883 & $<0.001$ \\
\hline
\end{tabular}

FPG, fasting plasma glucose; IDet, insulin detemir; NPH, neutral protamine Hagedorn.

IDet group and $11.99 \mathrm{~kg}$ in NPH group). In addition, no significant difference in the treatment of neonatal safety endpoints between both groups were observed (table 5).

Our study also recorded the adverse drug reactions and incidence of hypoglycemia in both groups. As shown in table 5, the incidence of hypoglycemia was significantly lower in the IDet group than that in the NPH group $(\mathrm{P}=0.017)$. In the IDet group, only 14 patients $(11.67 \%)$ had $\geq 1$ hypoglycemia event during the treatment period, which often occurred between 11:00 and 12:00 before lunch without nocturnal hypoglycemia; NPH group had 28 patients (23.33\%) had $\geq 1$ hypoglycemic event, among which eight events occurred between 02:00 and 03:00, seven occurred before breakfast, 13 occurred before lunch, and one patient had frequent hypoglycemia. There was no difference in the incidence of adverse drug reactions, such as local swelling and itching between both groups $(\mathrm{P}=0.652)$.

\section{DISCUSSION}

In China, both patients and obstetricians are worried about the safety of insulin, especially the use of longacting insulin during pregnancy. Our study investigated the efficacy and safety of IDet versus NPH in treatment of gestational diabetes in order to provide evidence for the management of diabetes with IDet during pregnancy. Compared with NPH, our study found that: (1) IDet had a more obvious hypoglycemic effect, FPG compliance and fewer injections; and (2) IDet significantly reduced the incidence of hypoglycemia without increasing the incidence of adverse pregnancy outcomes and drug reactions.

NPH is one of the most commonly used intermediateacting insulin in the clinics. Before the safety of long-acting insulin is confirmed, NPH was often recommended as a basal insulin for pregnant women with diabetes. ${ }^{13}$ With the introduction of long-acting insulin analogues, such as insulin glargine and IDet into clinical practice, their safety and effectiveness are urgently needed to be proven. However, there is a lack of convincing evidence in this area. The largest randomized study evaluating the efficacy and safety of IDet in pregnancy versus NPH, both with prandial insulin aspart, was conducted in 310 women with Type 1 Diabetes Mellitus (T1DM) and concluded that IDet was non-inferior to NPH in type 1 diabetes pregnant women. ${ }^{14}{ }^{15}$ As for Type 2 Diabetes Mellitus (T2DM), the current evidence for the use of IDet is scarce. A retrospective cohort study of 91 women with GDM who were treated with either glyburide $(n=62)$ or IDet (29) showed that IDet had a better outcomes. Recently, a randomized trial recruited both GDM patients and pregnant women with T2DM to observe the efficacy and safety of IDet. ${ }^{16}$ The findings suggested that the efficacy of IDet in glycemic control was similar with NPH and hypoglycemic events were significantly lower when the short-acting insulin aspart was added. Consistently, our study showed that IDet was at least as effective as NPH in a long term of treatment when used as a basal insulin in pregnant women with diabetes. However, in the early period of treatment, IDet had a better performance than NPH in terms of controlling blood glucose. Probably, a larger sample size in our study may contribute to reveal differences of IDet and NPH in controlling blood glucose in early treatment than the study mentioned above. Although the total dose of IDet was higher than NPH because of less affinity of IDet than insulin NPH for the insulin receptor, ${ }^{17}$ our data revealed that IDet group had fewer injections than NPH group. This may be due to the unique hexameric form of IDet and its reversible combination with plasma albumin, which significantly prolonged the action time in patients. ${ }^{18}$ Moreover, IDet group also had a shorter length of stay in the hospital and reduced economic cost. Taken together, these improvements made the patients feel more satisfied and compliant when given a hypoglycemic therapy with IDet.

Table 4 Comparison of insulin need in IDet and NPH group (means \pm SD)

\begin{tabular}{llclc}
\hline Variable & IDet & NPH & T/Z & P value \\
\hline Dose (u/day ) & $12.32 \pm 5.05$ & $9.10 \pm 5.93$ & 4.523 & $<0.001$ \\
Novolin-R (u/day ) & $35.57 \pm 16.27$ & $33.11 \pm 14.23$ & 1.242 & 0.216 \\
Insulin (u/day ) & $47.89 \pm 18.94$ & $42.21 \pm 17.14$ & 2.430 & 0.016 \\
Frequency (t/day) & $3.74 \pm 0.78$ & $4.15 \pm 0.36$ & 5.65 & $<0.001$ \\
\hline
\end{tabular}

IDet, insulin detemir; NPH, neutral protamine Hagedorn. 
Table 5 Maternal and neonatal pregnancy outcomes in the two groups

\begin{tabular}{lllll}
\hline & IDet & NPH & T/ $\chi^{2}$ & P value \\
\hline Maternal pregnancy outcomes & & & & \\
Gestational age at delivery (weeks ) & $38.64 \pm 2.19$ & $38.15 \pm 3.05$ & 1.418 & 0.157 \\
\hline Cesarean section, $\mathrm{n}(\%)$ & $78(65.00)$ & $74(61.67)$ & 0.287 & 0.592 \\
\hline Gestational weight gain (kg ) & $12.21 \pm 3.84$ & $11.99 \pm 4.11$ & 0.618 & 0.662 \\
\hline Hypertension during pregnancy, $\mathrm{n}(\%)$ & $14(11.67)$ & $23(19.17)$ & 2.588 & 0.107 \\
\hline Polyhydramnios, $\mathrm{n}(\%)$ & $10(8.33)$ & $8(6.67)$ & 0.240 & 0.624 \\
\hline Infection, $\mathrm{n}(\%)$ & $15(12.50)$ & $18(15.00)$ & 0.316 & 0.574 \\
\hline Hypoglycemia in pregnant women, $\mathrm{n}(\%)$ & $14(11.67)$ & $28(23.33)$ & 5.657 & 0.017 \\
\hline Adverse drug reactions, $\mathrm{n}(\%)$ & $3(2.5)$ & $2(1.67)$ & 0.203 & 0.652 \\
\hline Neonatal pregnancy outcomes & & & & \\
\hline Congenital anomaly, $\mathrm{n}(\%)$ & $3(2.50)$ & $3(2.50)$ & 0.000 & 1.000 \\
\hline Preterm delivery, $\mathrm{n}(\%)$ & $15(12.50)$ & $18(15.00)$ & 0.316 & 0.574 \\
\hline Birth weight (g) & $3257.67 \pm 496.60$ & $3179.75 \pm 671.81$ & 1.022 & 0.308 \\
\hline Low birth weight, $\mathrm{n}(\%)$ & $5(4.17)$ & $8(6.67)$ & 0.732 & 0.392 \\
\hline Macrosomia, $\mathrm{n}$ (\%) & $10(8.33)$ & $10(8.33)$ & 0.000 & 1.000 \\
\hline Hyperbilirubinemia, $\mathrm{n}(\%)$ & $51(42.50)$ & $56(46.67)$ & 0.422 & 0.516 \\
\hline NRDS, $\mathrm{n}$ (\%) & $3(2.50)$ & $3(2.50)$ & 0.000 & 1.000 \\
\hline Neonatal hypoglycemia, $\mathrm{n}(\%)$ & $5(4.17)$ & $10(8.33)$ & 1.778 & 0.182 \\
\hline NICU admission, $\mathrm{n}(\%)$ & $35(29.17)$ & $38(31.67)$ & 0.177 & 0.674 \\
\hline
\end{tabular}

IDet, insulin detemir; NICU, Neonatal intensive care unit; NPH, neutral protamine Hagedorn; NRDS, Neonatal respiratory distress syndrome.

We also compared the pregnancy outcomes between both groups. In general, the incidence of adverse pregnancy outcomes for both the mothers and newborns were comparable between two groups, which suggested that IDet was as good as NPH in the aspect of safety. Our results were consistent with the findings reported by previous studies. ${ }^{15} 19$ The incidence of gestational hypertension was also lower in IDet group compared with NPH $(11.67 \%$ vs $19.17 \%$ ), although the between-group difference did not reach statistical significance. In addition, the drug reactions were similar in both groups except for hypoglycemia occurrence. The results showed that only $11.67 \%$ of patients in the IDet group had $\geq 1$ hypoglycemia event during the treatment which occurred between 11:00 and 12:00 whereas the incidence of hypoglycemia was much higher in NPH group with eight nocturnal hypoglycemia and one frequent hypoglycemia. Notably, there was no nocturnal hypoglycemia in the IDet group and hypoglycemia in all patients happened before lunch, when the patients were able to treat themselves and no severe cases were reported. However, in the NPH group, hypoglycemia occurred at night and day and was irregular. Patients could not predict the occurrence of hypoglycemia, especially nocturnal hypoglycemia which threatens the safety of the fetus. This can also explain why NPH group reached the ambitious targeted blood glucose slower since more cautious procedures were needed in adjusting (ie, increasing and decreasing) insulin doses used. Meanwhile, we also assessed neonatal hypoglycemia. It is well recognized that hypoglycemia occurs more frequently during pregnancy when given the intensive insulin treatment. ${ }^{20}$ Hence, one of the major concerns is to reduce the rate of hypoglycemia when patients are treated with insulin or insulin analogs. Similar to other insulin preparations, insulin detemir does not cross the human placenta at therapeutic doses. ${ }^{21}$ Our data demonstrated that the rate of neonatal hypoglycemia was not significantly lower with IDet compared with NPH group, but it was noted that fewer newborns experienced such an event in IDet group than NPH groups $(4.17 \%$ vs $8.33 \%$, respectively). These data suggested that IDet can be safely prescribed to women with diabetes during pregnancy.

High blood sugar can increase the risk of fetal malformations. Serious adverse outcomes (perinatal death and/ or congenital malformations) were associated with higher HbAlc levels before and during pregnancy. ${ }^{22}$ Furthermore, even small elevations in HbAlc in pregnant women with diabetes have been associated with significantly increased rates of congenital malformations. ${ }^{23}$ In this study, although there was no increased risk of malformations in both groups, it was worth noting that a total of six congenital malformations occurred in the newborns of both groups of patients and the total incidence of congenital malformations was 250 per 10000 , which was remarkable higher than that in 2011 (153.23 per 10000$).{ }^{24}$ Some studies suggested that if the embryo is subjected to the effects of the hyperglycemia environment before and after embryo implantation, the cell's own apoptotic program is prone to start, leading to multiple organ deformities. ${ }^{25}$ Therefore, even if 
the blood glucose reaches the targets, the rate of congenital malformations may not be significantly reduced. This suggested that the management of blood glucose before pregnancy or at early pregnancy may be more worthy of attention. Pregnant women who are at high risk of developing diabetes need to monitor blood sugar as early as possible in order to reduce the incidence of congenital malformations.

In conclusion, the use of both long-acting insulin IDet combined with short-acting insulin and NPH combined with short-acting insulin was shown to effectively control blood glucose levels in pregnancy with diabetes. IDet was much faster and more effective in controlling blood glucose with fewer injections. Furthermore, considering a lower risk of hypoglycemia and no increase in adverse pregnancy outcomes, insulin detemir is worthy of clinical promotion as an ideal basal insulin in pregnancy with diabetes. But considering the numbers of the adverse effects were small, more researches with larger sample size are needed to state that there is no difference in safety between the two treatments.

Acknowledgements We thank the personnel at the Northwest Women and Children's Hospital for the help of recruiting patients. Also, we thank all the participants for contribution to our study.

Contributors JJ, XL and ZHa designed the study, wrote the manuscript. JJ, ZHe, $\mathrm{NG}$ and $\mathrm{HZ}$ collected the data. ZHe and JG were involved in data cleaning and statistics. YM and ZM contributed to the interpretation of the results and critical revision of the manuscript for important intellectual content. XL and ZHa are the study guarantors. All authors contributed to data analysis, drafting and revising the article, gave final approval of the version to be published.

Funding This work was supported by the National Key Research and Development Plan of China (no. 2016YFC1000101).

Disclaimer The sponsor of the study was not involved in the design, conduct, or interpretation of the study.

Competing interests None declared.

Patient consent for publication Not required.

Ethics approval The protocol was approved by the ethical committee of Xi'an Jiaotong University (XJTU2012-076) and the Northwest Women and Children's Hospital (NWCH2012-012). All patients gave written informed consent. The principles of the Helsinki Declaration were followed.

Provenance and peer review Not commissioned; externally peer reviewed.

Data availability statement Data are available upon reasonable request.

Open access This is an open access article distributed in accordance with the Creative Commons Attribution Non Commercial (CC BY-NC 4.0) license, which permits others to distribute, remix, adapt, build upon this work non-commercially, and license their derivative works on different terms, provided the original work is properly cited, appropriate credit is given, any changes made indicated, and the use is non-commercial. See: http://creativecommons.org/licenses/by-nc/4.0/.

ORCID iD

Xiaogin Luo http://orcid.org/0000-0002-3572-3909

\section{REFERENCES}

1 Hod M, Kapur A, Sacks DA, et al. The International Federation of Gynecology and Obstetrics (FIGO) Initiative on gestational diabetes mellitus: A pragmatic guide for diagnosis, management, and care. International Journal of Gynecology \& Obstetrics 2015;131:S173-211.

2 Guariguata L, Linnenkamp U, Beagley J, et al. Global estimates of the prevalence of hyperglycaemia in pregnancy. Diabetes Res Clin Pract 2014;103:176-85.

3 International Diabetes Federation. IDF diabetes atlas. 8th Edition. Brussels, Belgium: International Diabetes Federation, 2017.

$4 \mathrm{Xu}$ T, Dainelli L, Yu K, et al. The short-term health and economic burden of gestational diabetes mellitus in China: a modelling study. BMJ Open 2017;7:e018893.

5 Hochberg Z, Feil R, Constancia M, et al. Child health, developmental plasticity, and epigenetic programming. Endocr Rev 2011;32:159-224.

6 Association MD. Clinical practice recommendations: gestational diabetes mellitus. Diabetes Care 2003;26:103.

7 Lambert K, Holt RIG. The use of insulin analogues in pregnancy. Diabetes Obes Metab 2013;15:888-900.

8 Toledano Y, Hadar E, Hod M. Pharmacotherapy for hyperglycemia in pregnancy - The new insulins. Diabetes Res Clin Pract 2018;145:59-66.

9 Monami M, Marchionni N, Mannucci E. Long-Acting insulin analogues versus NPH human insulin in type 2 diabetes: a metaanalysis. Diabetes Res Clin Pract 2008;81:184-9.

10 Monami M, Marchionni N, Mannucci E. Long-Acting insulin analogues vs. NPH human insulin in type 1 diabetes. A metaanalysis. Diabetes Obes Metab 2009;11:372-8.

11 Obstetrics Group, Chinese Medical Association Obstetrics and Gynecology Branch, Chinese Medical Association Perinatal Medicine Branch Pregnancy and Diabetes Collaboration Group. Guidelines for diagnosis and treatment of pregnancy and diabetes. Chinese Journal of Obstetrics and Gynecology 2014;49:561-9.

12 Lee GT, Satyan MT, Grothusen JD, et al. A retrospective study comparing outcomes in a midwestern US population after introduction of IADPSG guidelines for gestational diabetes. J Matern Fetal Neonatal Med 2019;32:67-72.

13 Kitzmiller JL, Block JM, Brown FM, et al. Managing preexisting diabetes for pregnancy: summary of evidence and consensus recommendations for care. Diabetes Care 2008;31:1060-79.

14 Mathiesen ER, Hod M, Ivanisevic M, et al. Maternal efficacy and safety outcomes in a randomized, controlled trial comparing insulin detemir with NPH insulin in 310 pregnant women with type 1 diabetes. Diabetes Care 2012;35:2012-7.

15 Hod M, Mathiesen ER, Jovanovič L, et al. A randomized trial comparing perinatal outcomes using insulin detemir or neutral protamine Hagedorn in type 1 diabetes. $J$ Matern Fetal Neonatal Med 2014;27:7-13.

16 Herrera KM, Rosenn BM, Foroutan J, et al. Randomized controlled trial of insulin detemir versus NPH for the treatment of pregnant women with diabetes. Am J Obstet Gynecol 2015;213:426.e1-426. e7.

17 Kurtzhals P, Schäffer L, Sørensen A, et al. Correlations of receptor binding and metabolic and mitogenic potencies of insulin analogs designed for clinical use. Diabetes 2000;49:999-1005.

18 Home P, Kurtzhals P. Insulin detemir: from concept to clinical experience. Expert Opin Pharmacother 2006;7:325-43.

19 Imbergamo MP, Amato MC, Amodeo R, et al. Use of basal insulin analog detemir in pregnant women with type 1 diabetes: a case-control retrospective study. Giornale Italiano di Ostetricia e Ginecologia 2012;34:491-8.

20 de Valk HW, Visser GH, pregnancy Iduring. Labour and delivery. Best Pract Res Clin Obstet Gynaecol 2011;25:65-76.

21 Suffecool K, Rosenn B, Niederkofler EE, et al. Insulin detemir does not cross the human placenta. Diabetes Care 2015;38:e20-1.

22 HAPO Study Cooperative Research Group, Metzger BE, Lowe LP, et al. Hyperglycemia and adverse pregnancy outcomes. $N$ Engl $J$ Med 2008;358:1991-2002.

23 Suhonen L, Hiilesmaa V, Teramo K. Glycaemic control during early pregnancy and fetal malformations in women with type I diabetes mellitus. Diabetologia 2000;43:79-82.

24 Ministry of Health of the People's Republic of China. Report on prevention and control of birth defects in China, 2012: 1-16.

25 Moley KH. Hyperglycemia and apoptosis: mechanisms for congenital malformations and pregnancy loss in diabetic women. Trends Endocrinol Metab 2001;12:78-82. 